\title{
Energy spectra and turbulence generation in the wake of magnetic obstacles
}

\author{
Saša Kenjereš \\ Department of Multi-Scale Physics, Faculty of Applied Sciences and J.M. Burgerscentre \\ for Fluid Dynamics, Delft University of Technology, Leeghwaterstraat 39, 2628 CB Delft, \\ The Netherlands
}

(Received 2 August 2012; accepted 22 October 2012; published online 29 November 2012)

\begin{abstract}
Numerical simulations and analysis of flow and heat transfer of an electrically conducting fluid past magnetic obstacles are reported. We studied the channel flow configuration with electrically and thermally insulated horizontal walls containing a single or multiple (two or three) magnetic dipoles. Different values of the interactive parameter $0 \leq \mathrm{N} \leq 50$ and with a fixed value of $\mathrm{Re}=10^{3}$ are simulated. Detailed insights into energy spectra and turbulence generation in the wake of magnetic obstacles are provided. Although the temperature is a passive scalar, strong dissimilarities between mechanisms of production of the turbulent kinetic energy and temperature variance are observed. The long-term averaged second moments of velocity and temperature revealed the presence of anisotropic turbulence and countergradient diffusion of turbulent heat fluxes. It is concluded that configurations with multiple magnetic dipoles can be utilized in practical applications where the local generation of turbulence and intensification of mixing and heat transfer are required. (C) 2012 American Institute of Physics. [http://dx.doi.org/10.1063/1.4767726]
\end{abstract}

\section{INTRODUCTION}

The interaction between flows of electrically conducting fluids and external electromagnetic fields plays a central role in numerous technological applications, Molokov et al. ${ }^{1}$ Some of the numerous examples include electromagnetic mixing of glass melt (Gopalakrishnan et al. ${ }^{2}$ ), electromagnetic braking in continuous casting of steel (Cukierski and Thomas ${ }^{3}$ ), new generation of flow meters (Vire et al., ${ }^{4}$ Kirpo et al. ${ }^{5}$ ), etc. In great number of industrial applications where the electrically conductive fluids are used (e.g., crystal growth, heat exchangers, latest generation of fusion reactors), heat transfer plays a central role. Generic examples also include fully developed turbulent channel flows subjected to uniform magnetic fields of different orientations (Brouillette and Lykoudis, ${ }^{6}$ Reed and Lykoudis, ${ }^{7}$ Shimomura, ${ }^{8}$ Lee and Choi, ${ }^{9}$ Kenjereš et al. ${ }^{10}$ Boeck et al. ${ }^{11}$ ) and electromagnetically driven multiscale isothermal shallow (Rossi et al. ${ }^{12,13}$ Lardeau et al. ${ }^{14}$ Rossi et al.,${ }^{15}$ Akkermans et al.,${ }^{16}$ Duran-Matute et al. ${ }^{17}$ ) or deep (Kenjereš et al. ${ }^{18}$ ) layers with heat transfer (Kenjereš ${ }^{19-21}$ ).

The flow of electrically conducting fluids where there is an internal blockage can be generally divided into two categories. The first category includes flows past solid internal obstacles subjected to external uniform magnetic field. The second category includes flows in channels or ducts locally subjected to a localized (non-uniform) magnetic fields. The latter type are called magnetic obstacles. First, we will provide a short overview of some of the most recent studies dealing with these two categories of magnetohydrodynamics (MHD) flows.

Starting from the pioneering work of Hunt and Ludford ${ }^{22}$ and Kolesnikov and Tsinober, ${ }^{23}$ many analytical, numerical, and experimental studies in the literature have dealt with flows of electrically conducting fluids around non-conducting solid obstacles of different cross-area and subjected to a magnetic field of different orientations and strength. In some of the more recent studies, Mutschke et $a l .{ }^{24}$ presented a numerical analysis of two- and three-dimensional instabilities for a circular 
cylinder wake with an externally imposed uniform magnetic field aligned with the flow direction. In that research paper, a curve of neutral two-dimensional stability was shown for a range of Reynolds $(50 \leq \operatorname{Re} \leq 300)$ and interactive $(0 \leq \mathrm{N} \leq 0.4)$ numbers. It was demonstrated that a periodic vortex shedding flow motion was replaced with steady flow patterns for sufficiently strong N. In their follow-up study, Mutschke et al. ${ }^{25}$ provided more detailed insights on different scenarios of three-dimensional instabilities for an identical setup. Mück et al. ${ }^{26}$ studied three-dimensional MHD flows in rectangular ducts with a square cylinder. They considered relatively low values of the Reynolds number of $\mathrm{Re}=200$ and 250 under different strengths of a vertically imposed uniform magnetic field $(0 \leq \mathrm{Ha} \leq 36)$. For a pure hydrodynamic case $(\mathrm{Ha}=0)$, the flow exhibited characteristic intermittent pulsations of the drag and lift forces on the cylinder, and the appearance of the three-dimensional instabilities was confirmed. With an applied uniform magnetic field aligned with the square cylinder, a quasi-two-dimensional flow was generated even for relatively low values of interactive parameter $\mathrm{N}=1$. With further increase of the imposed magnetic field, it was possible to almost completely suppress the vortex shedding behind the cylinder. Dousset and Pothérat ${ }^{27}$ performed two-dimensional numerical studies of a liquid metal flow behind a circular cylinder with a vertically imposed magnetic field (parallel to the cylinder axis) for $\mathrm{Re} \leq 5000$ and $0 \leq \mathrm{Ha} \leq 2160$. The stability regime diagram was presented, including four characteristic regimes: the creeping flow regime, the steady symmetric attached recirculation regions, the laminar periodic flow regime (regular Kármán vortex street), and finally, the flow regime where secondary vortices are released from the side walls. Hussam et al. ${ }^{28}$ analyzed dynamics and heat transfer in a quasi-two-dimensional MHD flow past a circular cylinder $(50 \leq \mathrm{Re} \leq 3000)$ in a duct subjected to strong uniform vertical magnetic field $(0 \leq \mathrm{Ha} \leq 1200)$. It was observed that augmentation of heat transfer (up to twofold) occurred with an increase of blockage ratio (a ratio between circular cylinder diameter and the channel spanwise extension).

The numerical simulations of vorticity generation in a two-dimensional creeping flow ( $\mathrm{Re}$ $=0.05)$ past a magnetic obstacle $(0 \leq \mathrm{Ha} \leq 100)$ were addressed in Cuevas et al. ${ }^{29}$ The same authors extended their analysis to higher Reynolds numbers, $\mathrm{Re}=100$ and 200, keeping the identical magnetic field strength as in the previous study $(0 \leq \mathrm{Ha} \leq 100)$, Cuevas et al. ${ }^{30}$ It was observed that a wake behind the magnetic obstacle was formed. The wake becomes unstable after reaching a critical value of Ha. In a series of publications, Votyakov et al. ${ }^{31,32}$ and Votyakov and Kassinos ${ }^{33,34}$ addressed the structure of the wake of a magnetic obstacle in great detail. It was demonstrated that stationary flow patterns behind and within a magnetic obstacle exhibited considerably more complex patterns than in the wake of a solid obstacles. The most complex topology of flow included three pairs of vortices - magnetic (within the magnetic obstacle), connecting (in the near wake), and attached (in the far wake), Votyakov et al. ${ }^{31}$ The stability diagram was presented for a range of $100 \leq \operatorname{Re} \leq 900,0 \leq \mathrm{N} \leq 100$ and geometrical constrainment parameter $0.1 \leq \kappa \leq 1$ (defined as the ratio between magnetic spanwise extension and the spanwise channel size). Vortex shedding past a magnetic obstacle was observed for $\mathrm{Re}=900$ and $\mathrm{N}=9$, Votyakov and Kassinos. ${ }^{33}$ Beltran et $a l .{ }^{35}$ presented theoretical and numerical studies of a two-dimensional flow that was produced by imposing oscillation of a localized magnetic field in initially quiescent viscous and electrically conducting fluid. Kenjereš et al. ${ }^{36}$ presented numerical simulations and analysis of vortical structures behind a magnetic obstacle in transitional flow regimes $(100 \leq \mathrm{Re} \leq 900,4 \leq \mathrm{N} \leq 25)$. The second-order moments of velocity fields were shown for the very first time for $\operatorname{Re}=900$ and $3 \leq \mathrm{N}$ $\leq 25$.

The aim of the present work is to study the instantaneous and long-term averaged flow and heat transfer features of a three-dimensional MHD flow past different magnetic dipole configurations in a channel with electrically and thermally insulated horizontal walls, over a range of working parameters, but with a fixed inflow condition of $\operatorname{Re}=10^{3}$.

\section{MATHEMATICAL EQUATIONS}

The motion and heat transfer of an electrically conducting fluid subjected to an external magnetic field can be described by conservation equations of mass, momentum, energy, electric current density, 
and magnetic field, as follows:

$$
\begin{gathered}
\nabla \cdot \mathbf{v}=0, \quad \nabla \cdot \mathbf{b}=0, \quad \nabla \cdot \mathbf{j}=0 \\
\rho\left[\frac{\partial \mathbf{v}}{\partial t}+(\mathbf{v} \cdot \nabla) \mathbf{v}\right]=-\nabla p+\mu \nabla^{2} \mathbf{v}+\mathbf{j} \times \mathbf{b}, \\
\mathbf{j}=\sigma(-\nabla \phi+\mathbf{v} \times \mathbf{b}), \\
\nabla^{2} \phi=\nabla \cdot(\mathbf{v} \times \mathbf{b}), \\
\rho\left[\frac{\partial \theta}{\partial t}+(\mathbf{v} \cdot \nabla) \theta\right]=\frac{\mu}{\operatorname{Pr}} \nabla^{2} \theta,
\end{gathered}
$$

where $\mathbf{v}$ is the velocity, $\mathbf{b}$ is the magnetic field, $\mathbf{j}$ is the electric current density, $p$ is the pressure, $\phi$ is the electric potential, $\theta$ is the temperature. The last term in the momentum equation (Eq. (2)) represents the Lorentz force $\left(\mathbf{f}^{\mathbf{L}}=\mathbf{j} \times \mathbf{b}\right)$. The electric current density equation is obtained from Ohm's law in the moving frame of reference, Eq. (3). Taking the divergence of Ohm's law, the equation for electric potential is obtained, Eq. (4). In the thermal energy equation, Eq. (5), the viscous dissipation and Joule heating contributions are neglected.

The spatial distribution of the magnetic field is calculated from a semi-analytical expression originating from Biot-Savart's law and can be written as (Votyakov et $a l .{ }^{32}$ ),

$$
b_{\gamma}(x, y, z)=\frac{1}{\left|b_{0}\right|_{\mathrm{MC}}} \sum_{i, j, k= \pm 1}(i j k) \operatorname{arctanh}\left(f_{\gamma}\right),
$$

where $\left|b_{0}\right|_{\mathrm{MC}}$ is the magnetic field strength in the center of the magnetic dipole, $\gamma=x, y, z$ are magnetic field components, with $f_{\gamma}$ functions as

$$
\begin{array}{r}
f_{x}=\frac{y-j \frac{\mathrm{My}}{2}}{r(i, j, k)}, \quad f_{y}=\frac{x-i \frac{\mathrm{Mx}}{2}}{r(i, j, k)}, \\
f_{z}=\frac{\left(z-k \frac{\mathrm{Mz}}{2}\right) r(i, j, k)}{\left(x-i \frac{\mathrm{Mx}}{2}\right)\left(y-j \frac{\mathrm{My}}{2}\right)},
\end{array}
$$

and where distance parameter $r$ is calculated from

$$
r(i, j, k)=\left[\left(x-i \frac{\mathrm{Mx}}{2}\right)+\left(y-j \frac{\mathrm{My}}{2}\right)+\left(z-k \frac{\mathrm{Mz}}{2}\right)\right]^{1 / 2} .
$$

The characteristic magnetic geometry parameters (Mx, My, Mz) are sketched in Fig. 1. The characteristic fluid properties are $\rho$ - the density, $\mu$ - the dynamic viscosity, $\sigma$ - the electric conductivity, and $\operatorname{Pr}-$ the $\operatorname{Prandtl}$ number $(\operatorname{Pr}=v / a$, where $v$ is the kinematic viscosity, and $a$ is thermal diffusivity, respectively).

\section{NON-DIMENSIONAL PARAMETERS}

The working fluid is selected to mimic galinstan, an eutectic alloy consisting of gallium, indium, and tin, with a typical composition of $68.5 \% \mathrm{Ga}, 21.5 \% \mathrm{In}$, and $10 \% \mathrm{Sn}$. The typical properties of galinstan are $\rho=6360 \mathrm{~kg} / \mathrm{m}^{3}, \mu=1.1624 \times 10^{-3} \mathrm{~Pa} \mathrm{~s}, \sigma=3.36 \times 10^{6} \mathrm{~S} / \mathrm{m}$ and $\mathrm{Pr}$ $=0.022$. By selecting the characteristic length-scale to be the half-channel height $\left(\mathrm{H}_{0}=\mathrm{H} / 2\right)$ and the characteristic velocity to be the averaged fully developed laminar parabolic profile $\left(\mathrm{V}_{0}\right)$, the following set of non-dimensional parameters (and their ranges) can be written as

$$
\operatorname{Re}=\frac{V_{0} \mathrm{H}_{0}}{v} \quad\left(\operatorname{Re}=10^{3}\right),
$$




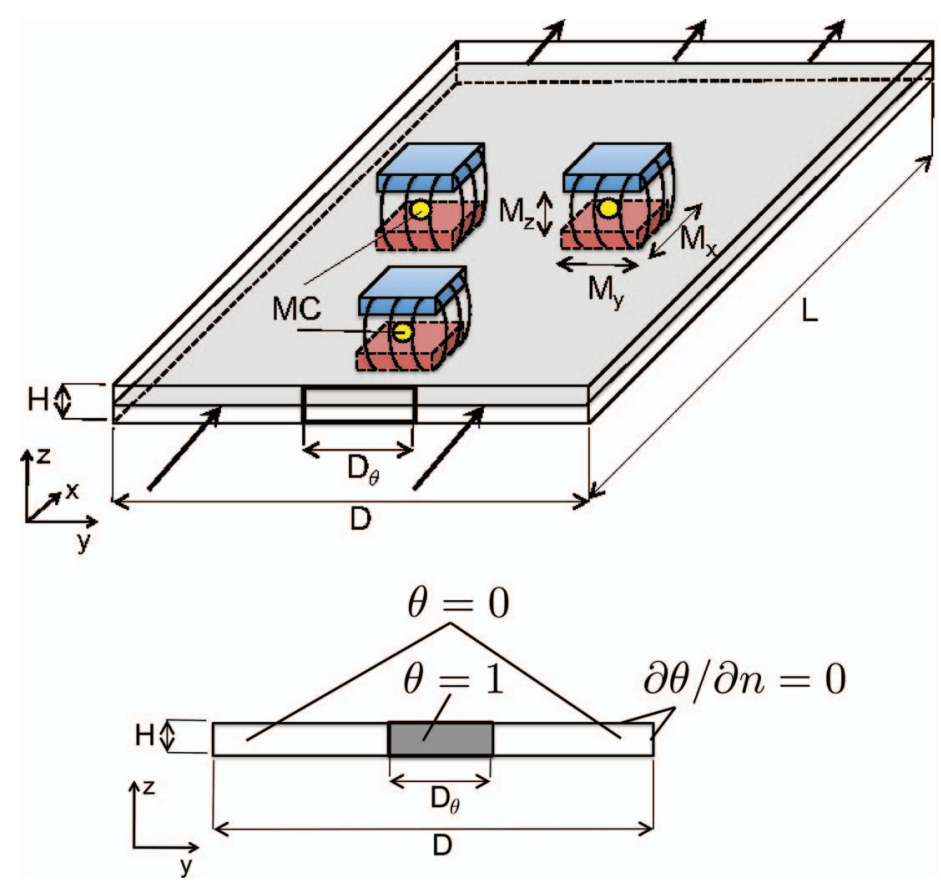

FIG. 1. (Top) a non-isothermal flow entering a channel with adiabatic walls is considered. Sketch of one of the geometries (three magnetic dipoles) is shown. The depth, length, and height of the domain are indicated by $\mathrm{D}, \mathrm{L}$, and $\mathrm{H}$, respectively. Here, L: D: $H=1: 0.3: 0.02$. The permanent magnets of opposite polarities $(\mathrm{N}, \mathrm{S})$ are indicated by red and blue blocks with $M_{x}: M_{y}: M_{z}=0.03: 0.04: 0.03$, where $M_{x}$ and $M_{y}$ are the length and depth, whereas $M_{z}$ is the vertical distance between magnets of opposite polarities. The locations of the magnetic dipoles centers are indicated by $\mathrm{MC}\left(\mathrm{x}_{\mathrm{MC}}, \mathrm{y}_{\mathrm{MC}}, \mathrm{z}_{\mathrm{MC}}\right)$. (Bottom) the inlet temperature and boundary conditions. Here, $\mathrm{D}_{\theta}: \mathrm{D}=0.04: 0.3$. All characteristic dimensions are in meters $(\mathrm{m})$.

$$
\begin{gathered}
\operatorname{Pr}=\frac{v}{a} \quad(\operatorname{Pr}=0.022), \\
\mathrm{N}=\frac{\sigma\left|b_{0}\right|^{2} \mathrm{H}_{0}}{\rho V_{0}} \quad(0 \leq \mathrm{N} \leq 50),
\end{gathered}
$$

where the $\left|b_{0}\right|$ is the magnetic dipole strength in the central horizontal plane $(\mathrm{z} / \mathrm{H}=0.5)$. The Hartmann number is also often used, which is defined as

$$
\mathrm{Ha}=(\operatorname{ReN})^{1 / 2} \quad(0 \leq \mathrm{Ha} \leq 224) .
$$

\section{NUMERICAL METHOD}

The system of Eqs. (1)-(5) is discretised by using a finite volume Navier-Stokes solver for general non-orthogonal structured geometries, Kenjereš and Hanjalić, ${ }^{37-40}$ Kenjereš. ${ }^{41}$ The Cartesian vector and tensor components in the non-staggered grid arrangements are applied, i.e., all variables are located in the centers of hexahedral control volumes (CVs). The SIMPLE algorithm is used for coupling between velocity and pressure fields. The diffusive terms of both momentum and thermal energy equations are discretised by a second-order central differencing scheme. This scheme is also used for the convective terms of the momentum equation. The convective term of the thermal energy equation is discretised by a total-variation-diminishing scheme with the min-mod flux limiter, Roe. ${ }^{42}$ The time integration is performed by a fully implicit second-order scheme that employs three consecutive time steps. The three-dimensional domain-decomposition technique with message passing interface directives is used for execution of the code on multiple processors. 
TABLE I. Central locations of magnetic dipoles (centers of magnetic gap between permanent magnets at top and bottom horizontal walls).

\begin{tabular}{lccc}
\hline \hline Configuration: & $\mathrm{x}_{\mathrm{MC}}[\mathrm{m}]$ & $\mathrm{y}_{\mathrm{MC}}[\mathrm{m}]$ & $\mathrm{z}_{\mathrm{MC}}[\mathrm{m}]$ \\
\hline One dipole & 0.065 & 0.15 & 0.01 \\
Two dipoles & 0.065 & 0.1 & 0.01 \\
& 0.065 & 0.2 & 0.01 \\
Three dipoles & 0.065 & 0.15 & 0.01 \\
& 0.15 & 0.1 & 0.01 \\
& 0.15 & 0.2 & 0.01 \\
\hline \hline
\end{tabular}

\section{RESULTS AND DISCUSSION}

\section{A. Setup and boundary conditions}

A sketch of one of the geometries considered is shown in Fig. 1. The locations of the permanent magnets, which form the magnetic dipoles, are indicated by red and blue blocks. The centers of the magnetic dipoles in the central horizontal plane $(\mathrm{z} / \mathrm{H}=0.5)$ are indicated by $\mathrm{x}_{\mathrm{MC}}, \mathrm{y}_{\mathrm{MC}}$, and $\mathrm{Z}_{\mathrm{MC}}$ and are listed in Table I for all situations considered. The fully developed laminar profiles are imposed at the inlet $(\mathrm{y}-\mathrm{z})$ plane $(\mathrm{x}=0 \mathrm{~m})$ with $\mathrm{Re}=10^{3}$. The inlet temperature distributions are selected to mimic the delta distribution as $\theta^{*}=0$ for $0 \leq y<\left(\mathrm{D} / 2-\mathrm{D}_{\theta} / 2\right)$ and $\left(\mathrm{D} / 2+\mathrm{D}_{\theta} / 2\right)<y$ $\leq \mathrm{D}$, whereas $\theta^{*}=1$ for $\left(\mathrm{D} / 2-\mathrm{D}_{\theta} / 2\right) \leq y \leq\left(\mathrm{D} / 2+\mathrm{D}_{\theta} / 2\right)$. Here, $\theta^{*}=\theta / \Delta \theta$ and $\Delta \theta=1$. The bottom- and top-boundaries of the domain are treated as thermally and electrically insulated walls ( $u=v=w=0, \partial \theta / \partial n=0, \partial \phi / \partial n=0$, where "n" is the wall-normal direction). The outlet (y-z) plane is treated as a convective boundary condition for all variables, i.e., $\partial / \partial t+V_{0} \partial / \partial x=0$. The side boundaries are treated as a free-slip for velocity $(\partial u / \partial n=0, v=w=0)$ and a zero-gradient boundary condition is imposed for temperature and electric potential $(\partial \theta / \partial n=0, \partial \phi / \partial n=0)$. A non-uniform numerical mesh with $(\mathrm{nx}, \mathrm{ny}, \mathrm{nz})=(462,162,102) \approx 7.6 \times 10^{6} \mathrm{CVs}$, refined in the proximity of the upper and lower horizontal walls and in the proximity of the magnetic dipoles, is employed for all simulations. A grid expansion factor of 1.1 is employed in these regions. Within the magnetic obstacles, 40 control volumes are employed in each horizontal direction. The Kolmogorov length scales are estimated as $\eta=\left(v^{3} / \varepsilon\right)^{1 / 4}$, where the dissipation rate of the turbulent kinetic energy is approximated as $\varepsilon \approx \mathrm{V}_{0}^{3} / \mathrm{H}_{0}$, giving $\eta \approx 6 \times 10^{-5} \mathrm{~m}$. The numerical mesh employed satisfies this requirement in the proximity of the horizontal walls, within the magnetic dipoles, and in the nearwake regions. Farther away it is approximately $2 \eta \leq \Delta V^{1 / 3} \leq 5 \eta$, where $\Delta V=\Delta x \cdot \Delta y \cdot \Delta z$ is a characteristic volume of the numerical computational cell. An overview of performed simulations is given in Table II.

\section{B. Instantaneous flow features}

To illustrate similarities and to emphasize some significant differences between flows behind solid bluff-bodies and magnetic obstacles, characteristic velocity vectors, pressure- and

TABLE II. Overview of simulations considered. Note that a fully developed laminar inlet is assumed for all simulations, Re $=10^{3}$.

\begin{tabular}{lccc}
\hline \hline Configuration: & $\mathrm{N}[-]$ & Ha $[-]$ & $\left|b_{0}\right|$ MC $[\mathrm{T}]$ \\
\hline One dipole & 1 & 32 & 0.08 \\
& 5 & 71 & 0.18 \\
& 10 & 100 & 0.25 \\
& 50 & 224 & 0.56 \\
Two dipoles & 1 & 32 & 0.08 \\
& 10 & 100 & 0.25 \\
Three dipoles & 1 & 32 & 0.08 \\
& 10 & 100 & 0.25 \\
\hline \hline
\end{tabular}



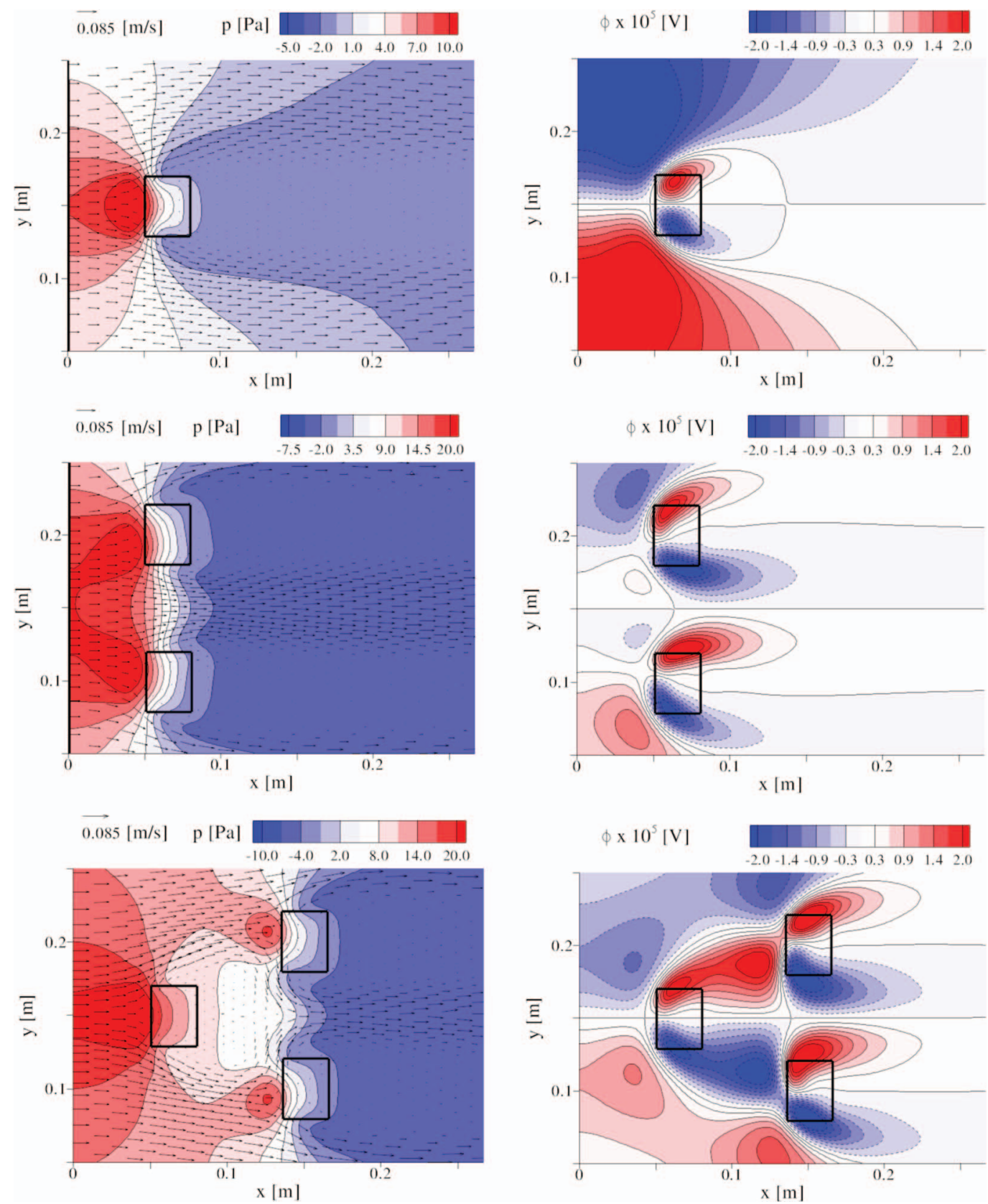

FIG. 2. A zoom-in of the pressure contours and velocity vectors (left) and of electric potential (right) of an instantaneous realization in the central horizontal plane in a channel flow of a conductor subjected to localized magnetic fields, $\operatorname{Re}=10^{3}$, $\mathrm{N}=10$. The locations of the magnetic dipoles projections in the central horizontal plane are depicted by black rectangles (varied in row).

electric-potential distributions in the central horizontal plane $(\mathrm{z} / \mathrm{H}=0.5)$ for the different magneticdipole configurations are shown in Fig. 2. Note how the velocity vectors are partially deflected from the magnetic-obstacle regions - similar to the case for the solid obstacles - but at the same time, a flow reorganization within the magnetic obstacles takes place (see continuous pressure distribution within the rectangle regions). This flow reorganization is a consequence of the Lorentz force action (Eq. (2)). Different flow structures within and behind a single magnetic obstacle are analyzed in more detail in Cuevas et al. ${ }^{29,30}$ Votyakov et al. ${ }^{31,32}$ Votyakov and Kassinos. ${ }^{33,34}$ It is found that for different values of the interactive parameter $\mathrm{N}$, the following scenarios can occur: flow is slightly 

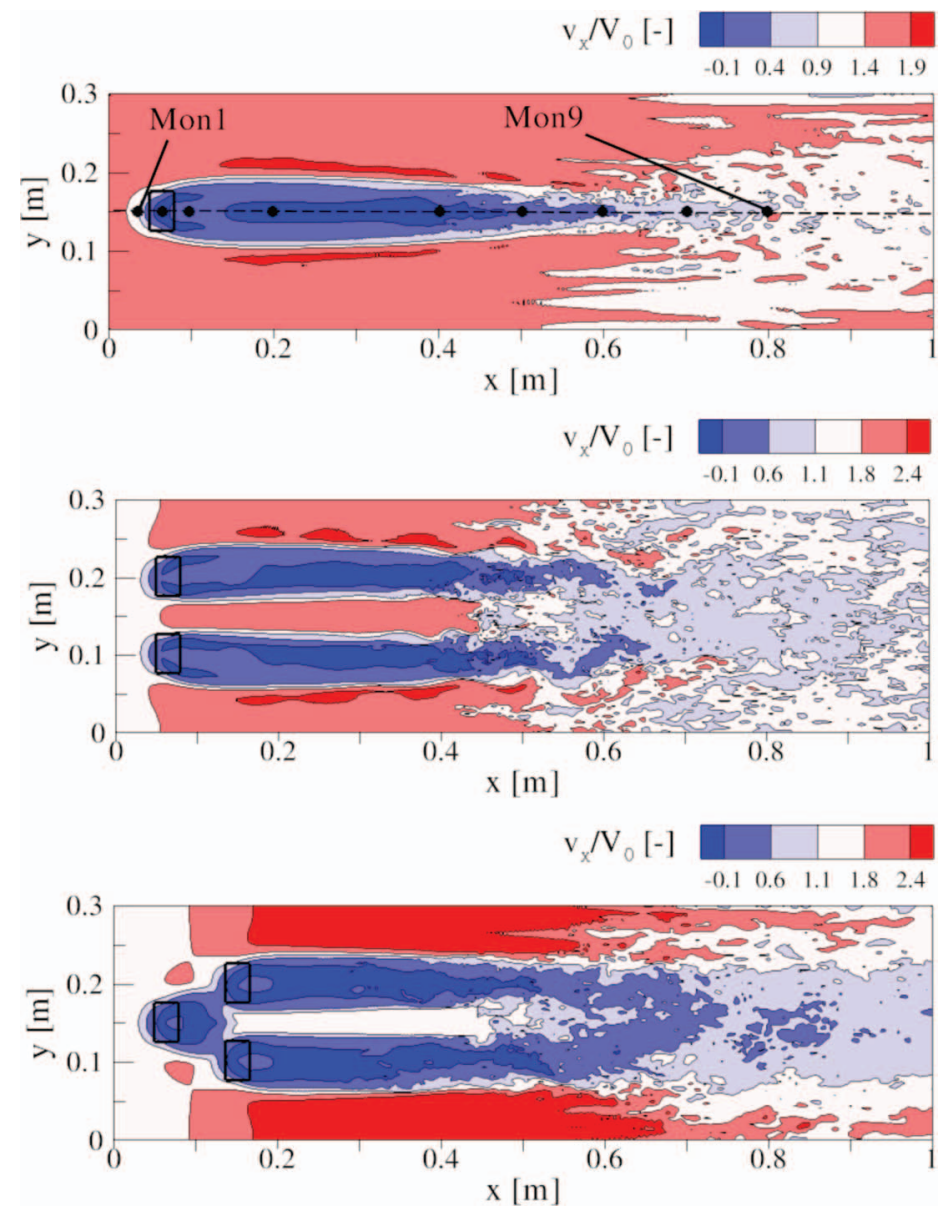

FIG. 3. Contours of the instantaneous non-dimensional horizontal velocity $\left(v_{x} / V_{0}\right)$ in the central horizontal plane for Re $=1000$ and $\mathrm{N}=10$, one magnetic dipole (top), two magnetic dipoles (middle), and three magnetic dipoles (bottom) configurations. The locations of characteristic monitoring locations in the central horizontal plane $(\mathrm{z}=0.01 \mathrm{~m})$ along $\mathrm{y}$ $=0.15 \mathrm{~m}$ line are also shown (Mon1-Mon9) (top). The horizontal locations of monitoring points (identical for all three configurations) are: $0.0375,0.065,0.1,0.2,0.4,0.5,0.6,0.7$, and $0.8 \mathrm{~m}$, respectively.

deflected and no recirculative patterns are observed (for low $\mathrm{N}$ ), a pair of the inner vortices is generated (for intermediate $\mathrm{N}$ ), and finally, three pairs of vortices are formed (for high $\mathrm{N}$ ), Votyakov et al. ${ }^{31}$ The introduction of the multi-magnetic dipoles makes it possible to impose additional flow reorganization. For example, for the configuration adopted here, with two magnetic dipoles, which are shifted in the spanwise (y) direction with respect to the domain centerline, the incoming flow accelerates similar to the "street canyon" effect for air flow between buildings, Fig. 2 (middle). The configuration with three magnetic dipoles superimposes a single magnetic dipole and two magnetic dipoles cases, which enables additional channeling of the wake regions. The contours of the electric potential are shown in Fig. 2 (right). Although the direct effects of the Lorentz force are confined in the magnetic obstacle regions, the fluctuations of the self-generated electric field $(\mathbf{e} \sim-\nabla \phi)$ are distributed throughout the entire flow by the diffusive mechanism, Eq. (4).

The most salient instantaneous flow features are shown in Fig. 3 where contours of the nondimensional horizontal velocity $v_{x} / V_{0}$ in the central horizontal plane are shown for three configurations at fixed $\mathrm{Re}=10^{3}$ and $\mathrm{N}=10$. The intermediate value of the interactive parameter $\mathrm{N}$ $=10$ is sufficient to generate adequate braking of the flow and to produce recirculative wake regions (identified as regions with negative values of the streamwise velocity). For the situation of two magnetic dipoles, two characteristic wakes are separated by a high-velocity region in between, Fig. 3 (middle). In contrast to this, for the case of three magnetic dipoles, the first obstacle deflects and 
slows down the flow in the central part of the domain, such that the wakes from the two remaining dipoles are separated by a region with lower streamwise velocity, Fig. 3 (bottom). The flow structures produced by the Kelvin-Helmholtz instability mechanism can be clearly seen in Fig. 3 (top and middle). They originate from the strong shear in the spanwise direction along the outer edge of the recirculative region. It can be concluded that by imposing different combinations of magnetic dipoles, the dynamics and structure of wake interactions can be significantly altered, Fig. 3 (middle and bottom).

The spatial distributions of the instantaneous temperature under identical conditions are shown in Fig. 4. It can be observed that the near-wake region is quite stable and that intermittent structures are mainly focused farther downstream in the far-wake region. These intermittent regions are triggered at $\mathrm{x} / \mathrm{L}=0.45$, for both two- and three-dipole configurations. For a single magnetic dipole case, the distinct intermittent regions are initialized at $x / L=0.6$. The configuration with three magnetic dipoles produces more intensive mixing in the spanwise direction, as seen from isosurfaces of instantaneous temperature, Fig. 4 (bottom).

\section{Energy spectra at characteristic locations}

The time-series of all dependent variables are collected at characteristic locations Mon1 - Mon9 along the domain centerline $(\mathrm{z}=0.01 \mathrm{~m}$ and $\mathrm{y}=0.15 \mathrm{~m})$ as shown in Fig. 3 (top). Note that the Mon 1 location is in front of the magnetic core region, the Mon2 exactly in the center of magnetic core, and remaining monitoring points (Mon3-Mon9) are behind the magnetic obstacle. Temporal energy spectra of the spanwise velocity, temperature, and electric potential are calculated at monitoring locations. Although the time series contains the complete history of time variations of variables, only parts of time series for which flow is fully developed and statistically converged, are used for calculation of the power spectra density (PSD) distributions. The discrete Fourier transformation is performed for all sets containing recordings of about $10^{4}$ time steps. Note that for $\mathrm{N}=1$ and all configuration studied, although the weak wake regions are created, the vortex shedding is not observed (i.e., the steady flow solutions are obtained).

For the single magnetic dipole case, the spectra at monitoring points for two values of $\mathrm{N}$ are shown in Fig. 5 (left) $(\mathrm{N}=5)$ and (right) $(\mathrm{N}=50)$. For both values of $\mathrm{N}$, identical distinct frequencies are obtained for the velocity and electric potential, as shown in Fig. 5 (top/bottom). This is expected since the velocity is in the source term in the Poisson equation of electric potential, Eq. (4). For $\mathrm{N}$ $=5$ case, the first distinct frequency of $f_{1}=0.24 \mathrm{~Hz}$ for $v_{y} / V_{0}$ and $\phi$ is present for monitoring points Mon1-Mon6, Fig. 5 (top/bottom). Note that this distinct frequency is already obtained for the Mon1 location, i.e., the flow starts to experience the presence of the magnetic field even before entering the magnetic dipole region due to the pressure field. The imprints of the secondary frequency $f_{2}$ $=0.105 \mathrm{~Hz}$ are present at Mon3-Mon7 for both $v_{y} / V_{0}$ and $\phi$. The third frequency $f_{3}=0.0095 \mathrm{~Hz}$ is visible at Mon1-Mon5 locations for $v_{y} / V_{0}$ and at Mon3-Mon9 for $\phi$. Note that this very low frequency is associated with slow flow structures, with typical time scales of 3-4 flow-through times. Finally, at Mon-1-Mon5 for $v_{y} / V_{0}$ and Mon1, Mon3, and Mon5 for $\phi$, the fourth distinct frequency is observed, $f_{4}=0.71 \mathrm{~Hz}$. The $f_{1}$ (for Mon1-Mon6) and $f_{2}$ (for Mon4-Mon6) are also observed for temperature spectra as well, Fig. 5 (middle). For the single magnetic dipole case at $\mathrm{N}=50$, two characteristic frequencies $f_{1}=1 \mathrm{~Hz}$ and $f_{2}=0.074 \mathrm{~Hz}$ are observed for the velocity and electric potential, Fig. 5 (right). For $\mathrm{N}=50$, the $f_{1}$ frequency is present at Mon1-Mon4 locations compared to Mon1-Mon6 locations for $\mathrm{N}=5$. The second frequency $f_{2}=0.074 \mathrm{~Hz}$ is consistently present for Mon3-Mon9 locations for $\left(v_{y} / V_{0}\right)$ and $\phi$, Fig. 5 (right/top and bottom). In contrast to the $\mathrm{N}=5$ case, for $\mathrm{N}$ $=50$, four distinct frequencies are identified for temperature, Fig. 5 (right/middle). In addition to $f_{1}$ $=1 \mathrm{~Hz}$ and $f_{2}=0.074 \mathrm{~Hz}$, also $f_{3}=0.148 \mathrm{~Hz}$ and $f_{4}=0.296 \mathrm{~Hz}$ are present. The $f_{3}=0.148 \mathrm{~Hz}$ frequency starts at Mon5 and is still present at Mon9.

The power spectra for the two and three magnetic dipole cases and $\mathrm{N}=10$ are shown in Fig. 6. It is interesting to see that for the case of two magnetic dipoles, there is only one distinct frequency, $f_{1}=0.54 \mathrm{~Hz}$ for the spanwise velocity and electric potential, Fig. 6 (left). This frequency can be observed only at Mon1-Mon6 locations. Under identical conditions, the temperature spectra shows three characteristic peaks, $f_{1}=0.54 \mathrm{~Hz}, f_{2}=1.055 \mathrm{~Hz}$, and $f_{3}=0.177 \mathrm{~Hz}$, but again, 


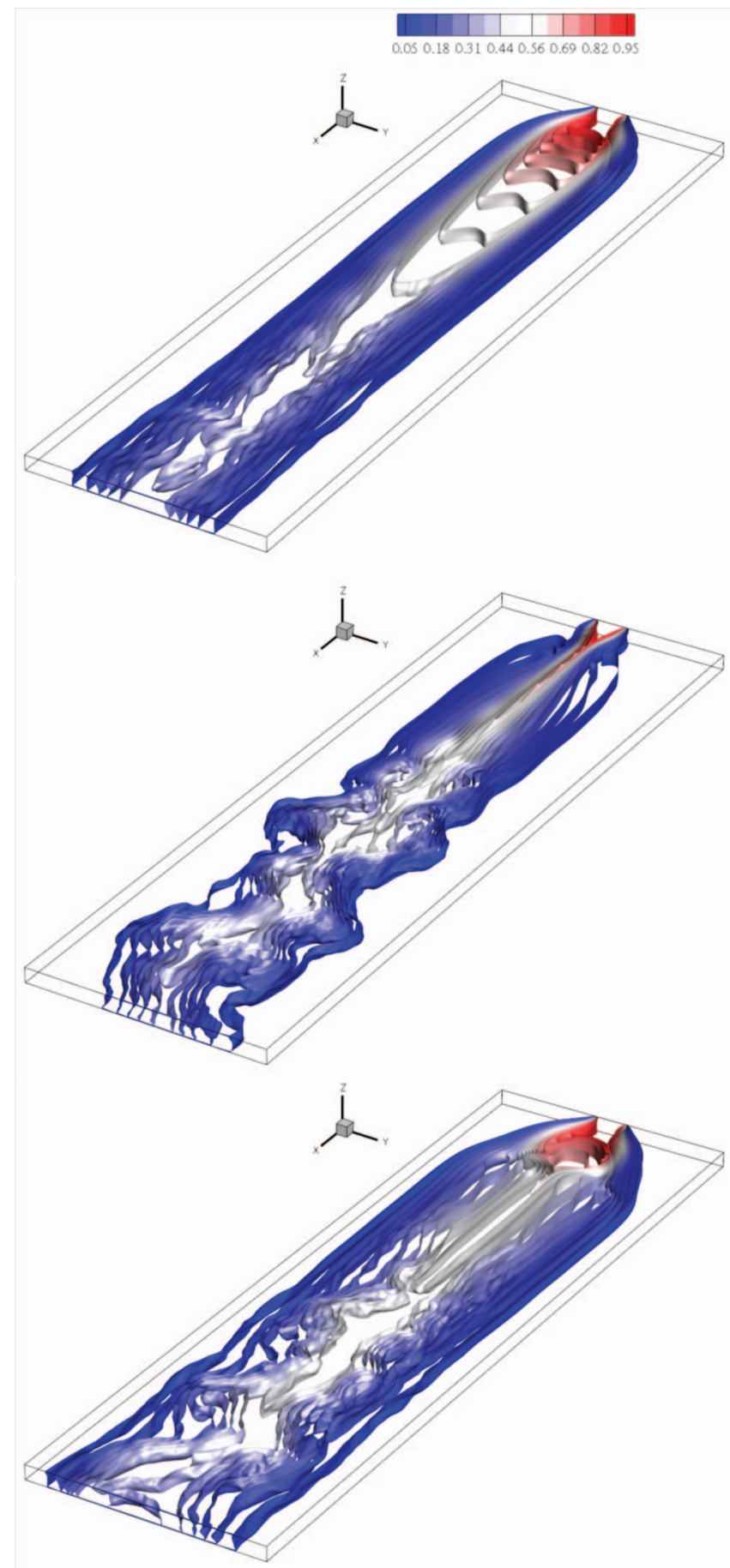

FIG. 4. Instantaneous isosurfaces of non-dimensional temperature $\theta^{*}=\theta / \Delta \theta$ (20 uniformly distributed levels) for $\operatorname{Re}=1000$ and $\mathrm{N}=10$, single magnetic dipole (top), two magnetic dipoles (middle), three magnetic dipoles (bottom) configurations. The flow is from top right to lower left.

only at Mon1-Mon4 locations. For the three-magnetic dipoles case and $\mathrm{N}=10$, two specific frequencies $f_{1}=1 \mathrm{~Hz}$ (Mon1-Mon4) and $f_{2}=0.064 \mathrm{~Hz}$ (Mon1-Mon9) are identified for $v_{y} / V_{0}$ and $\phi$, Fig. 6 (right). An additional three frequencies are distinguishable for the temperature, $f_{3}=0.127 \mathrm{~Hz}$ (Mon4-Mon9), $f_{4}=0.19 \mathrm{~Hz}$ (Mon8-Mon9), and finally, $f_{5}=0.226 \mathrm{~Hz}$ (Mon3-Mon4).

In our previous study, Kenjereš et al., ${ }^{36}$ where flows around a single magnetic obstacle in transitional regimes were shown, $\mathrm{Re}=900$ and $3 \leq \mathrm{N} \leq 25$, a single distinct frequency of the 
One Magnetic Dipole, $\mathrm{N}=5$
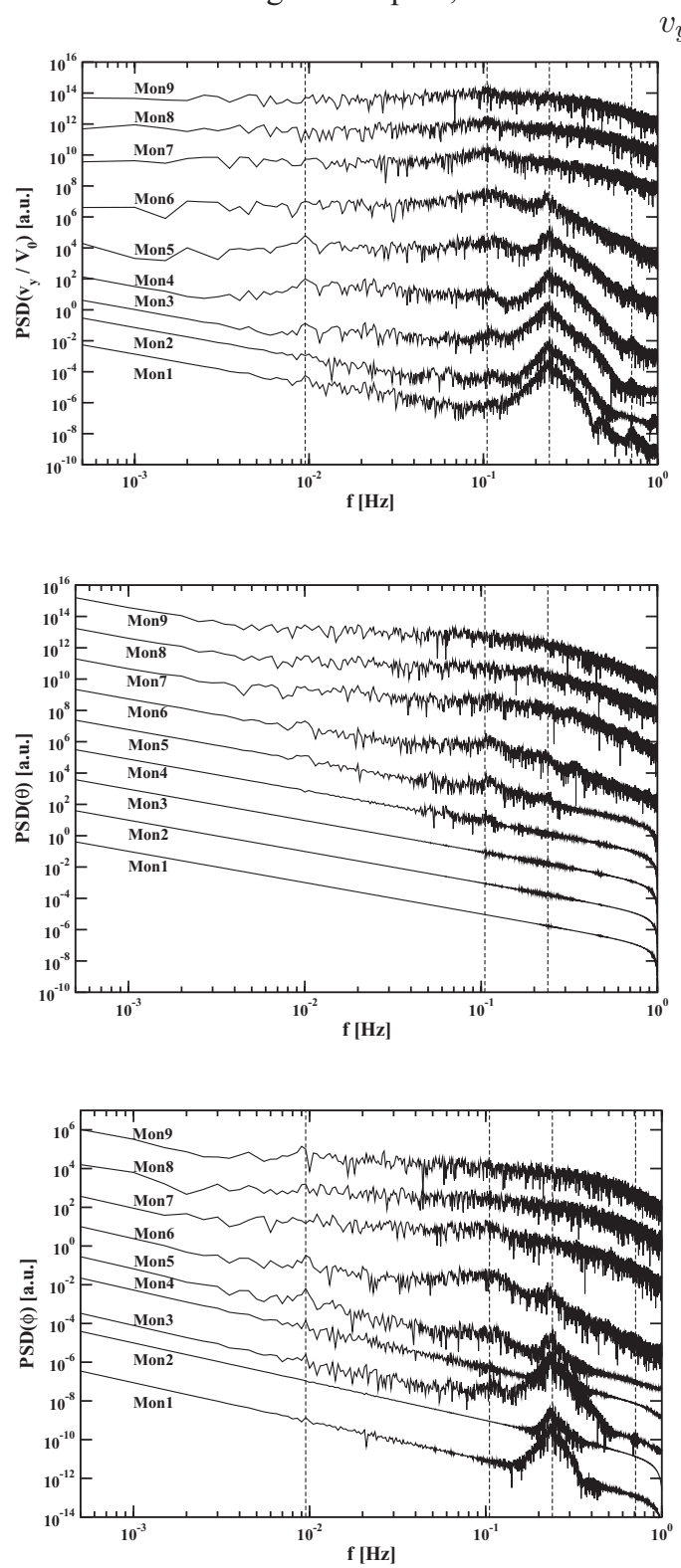

One Magnetic Dipole, N=50$$
v_{y} / V_{0}
$$

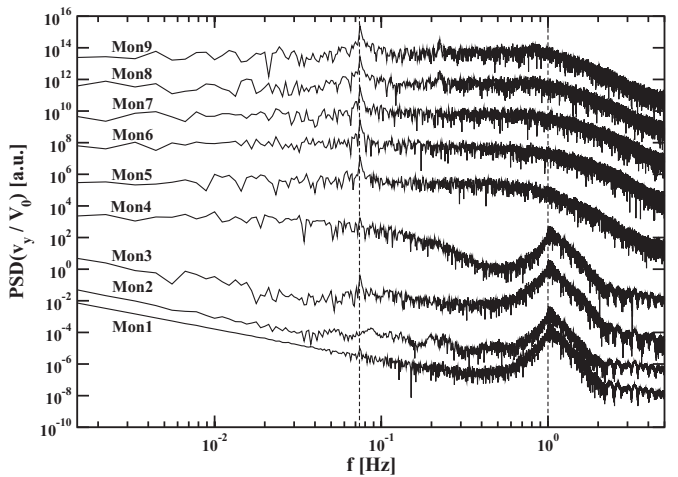

$\theta^{*}$

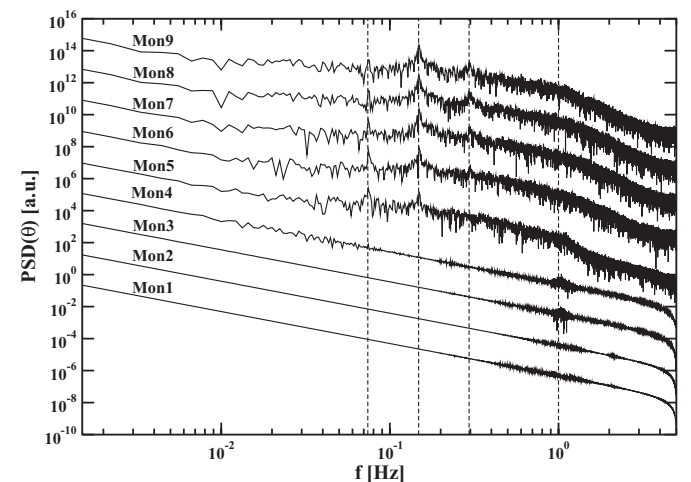

$\phi$

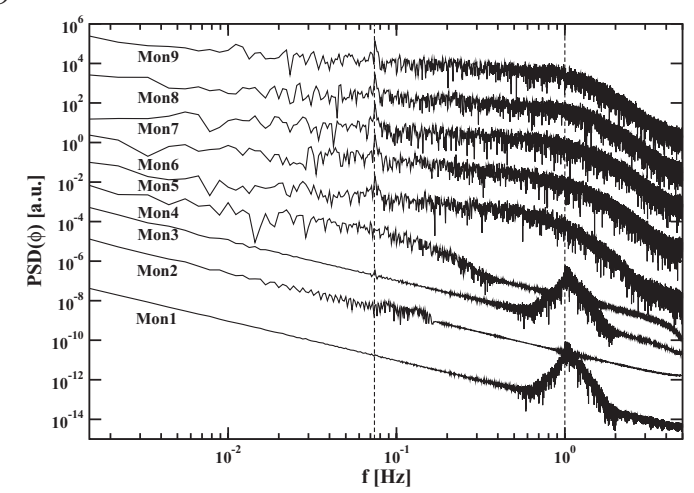

FIG. 5. Temporal energy spectra of the spanwise velocity $\left(v_{y} / V_{0}\right)$ (top), temperature $\left(\theta^{*}=\theta / \Delta \theta\right)$ (middle), and electric potential $(\phi)$ (bottom) for a single magnetic dipole configuration and magnetic interactive numbers of $\mathrm{N}=5$ (left row) and $\mathrm{N}=50$ (right row), respectively. The graphs for Mon2-Mon9 points are shifted vertically by incrementally increasing the scaling factor by $10^{2}$ between consecutive monitoring points. Note that dashed lines indicate the distinct key frequencies.

spanwise velocity of $f_{1}=0.11 \mathrm{~Hz}$ was observed for both $\mathrm{N}=3$ and 15 . This gave a value of the Strouhal (St) number of 0.144 (defined as $\mathrm{St}=\mathrm{f} \mathrm{M}_{\mathrm{y}} / V_{0}$ - where $\mathrm{M}_{\mathrm{y}}$ is the spanwise extension of the magnetic core). Elsewhere, Cuevas et al. ${ }^{29}$ reported $\mathrm{St}=0.1$, and Votyakov and Kassinos ${ }^{33}$ have given $\mathrm{St}=0.25$. This difference has been explained as resulting from the impacts of the side walls (two-dimensional versus three-dimensional geometry). In the present study, we observed multiple distinct frequencies, and consequently, a range of characteristic values of St can be calculated. For a single magnetic dipole and $\mathrm{N}=5$, we have $0.011 \leq \mathrm{St} \leq 0.282$. For same configuration but for $\mathrm{N}=50$, we obtained $0.087 \leq \mathrm{St} \leq 1.2$, i.e., significantly higher frequencies are recorded. A similar range of non-dimensional frequencies is obtained for two $(0.21 \leq \mathrm{St} \leq 1.24)$ and three $(0.075 \leq \mathrm{St}$ $\leq 1.22$ ) magnetic dipoles for $\mathrm{N}=10$. 
Two Magnetic Dipoles, $\mathrm{N}=10$

Three Magnetic Dipoles, N=10

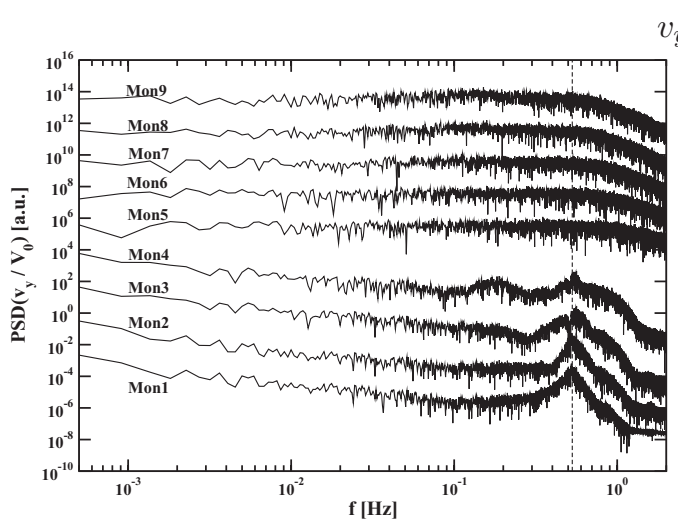

$v_{y} / V_{0}$

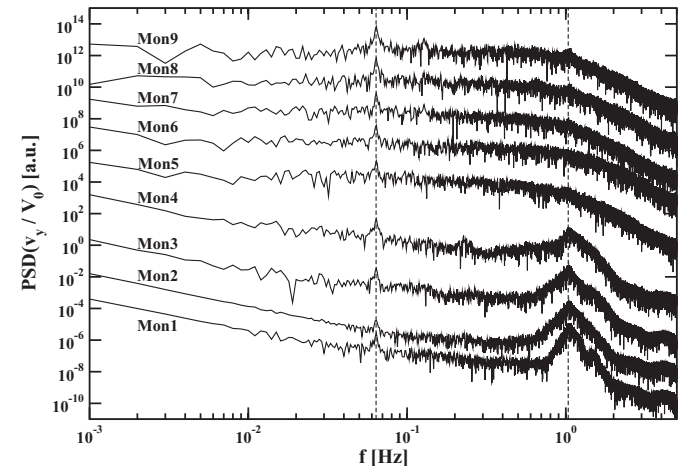

$\theta^{*}$
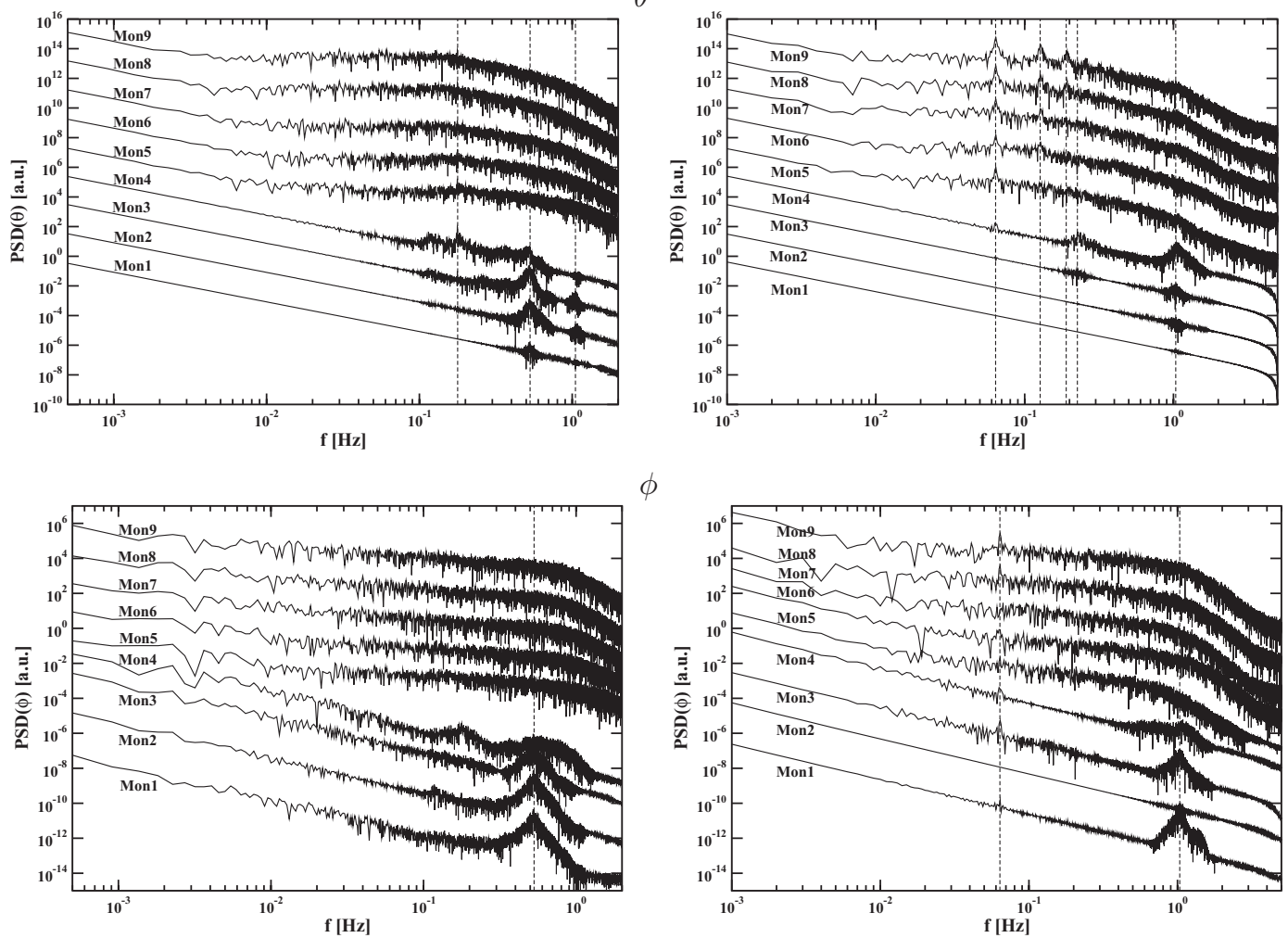

$\phi$

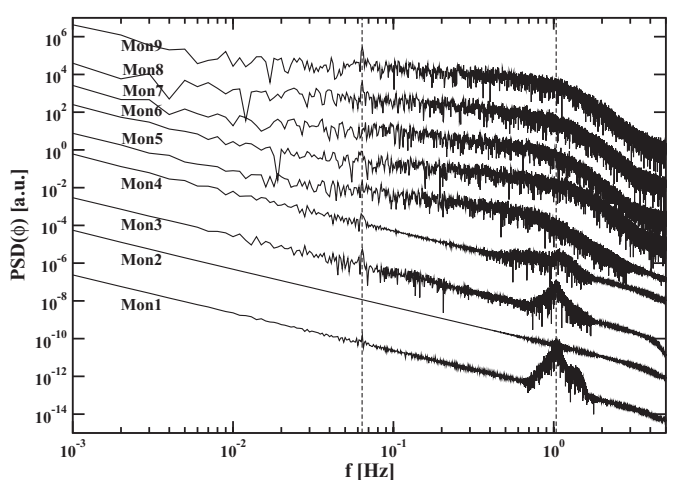

FIG. 6. Same as in previous figure only for 2- and 3-magnet configurations and $\mathrm{N}=10$.

\section{Long-term averaged turbulence fields}

Cuypers $e t a l{ }^{43}$ identified vortex bursts as a source of turbulence in a laminar environment. Since we are dealing with similar situations, i.e., a combination of the laminar inflow conditions and vortex bursts in wakes of magnetic obstacles, we will provide detailed insights into the turbulence production mechanisms. The long-term time averaging is performed for all instantaneous variables over a sufficiently long time interval that includes more than $10^{4}$ realizations, which corresponds to approximately 300 flow-through times $\left(t=L / V_{0}=1 / 0.034 \approx 30 \mathrm{~s}\right)$ for the domain considered, and where a typical value for a time-step of $\Delta t=0.1 \mathrm{~s}$ is used.

The contours of the turbulent kinetic energy production, which is defined as $P_{k}=-\overline{v_{i} v_{j}} \partial \overline{v_{i}} / \partial x_{j}$, where $\overline{v_{i} v_{j}}$ is the turbulent stress tensor, and $\overline{v_{i}}$ is the long-term averaged velocity, are shown in Figs. 7-9 (top). Similarly, the contours of the temperature variance (energy of the temperature 
One Magnetic Dipole, $\mathrm{N}=10$



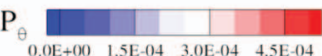

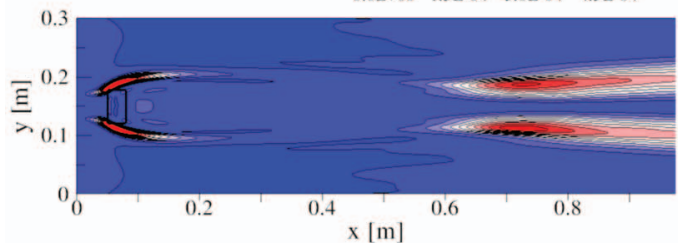

Two Magnetic Dipoles, $\mathrm{N}=10$

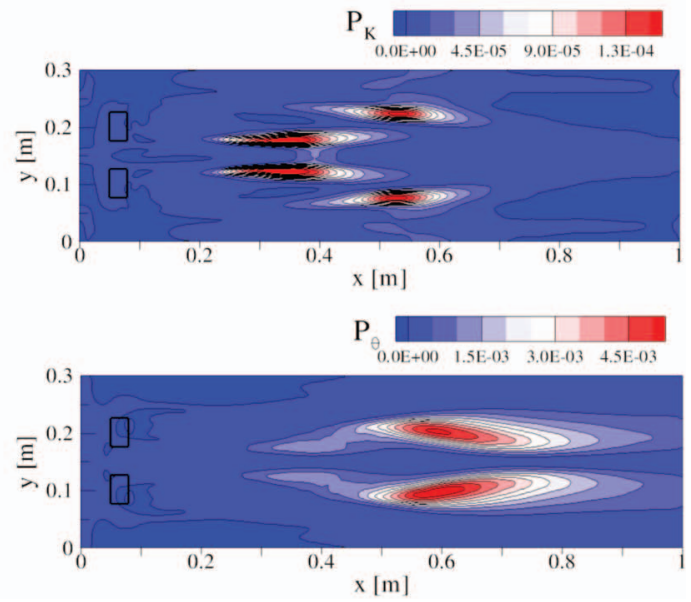

FIG. 7. Contours of the production of the turbulent kinetic energy $\left(\mathrm{P}_{k}\right)$ (top) and temperature variance $\left(\mathrm{P}_{\theta}\right)(\mathrm{bottom})$ in the central horizontal plane for the single-magnet (left) and two-magnet (right) configurations, $\operatorname{Re}=10^{3}, \mathrm{~N}=10$.

fluctuations) production, which is defined as $P_{\theta}=-\overline{\theta v_{i}} \partial \bar{\theta} / \partial x_{i}$, where $\overline{\theta v_{i}}$ is the turbulent heat flux, and $\bar{\theta}$ is the long-term averaged temperature, are shown in Figs. 7-9 (bottom). Under identical flow conditions, $\mathrm{Re}=10^{3}, \mathrm{~N}=10$, two and four distinct pockets of $P_{k}$ are generated in the central horizontal plane for one- and two-magnetic-dipoles configuration, respectively, Fig. 7 (top). The streamwise length of $\mathrm{P}_{k}$ pockets reduces from $0.4 \mathrm{~m}$ for the single magnetic dipole, to approximately half of size for the two-magnetic-dipoles configuration. The $P_{\theta}$ pockets exhibit significantly different behavior - with distinct structures generated at the edges of the magnetic region (for a single magnetic dipole case) and in distant wake regions (for both single- and two-magnet dipoles cases),
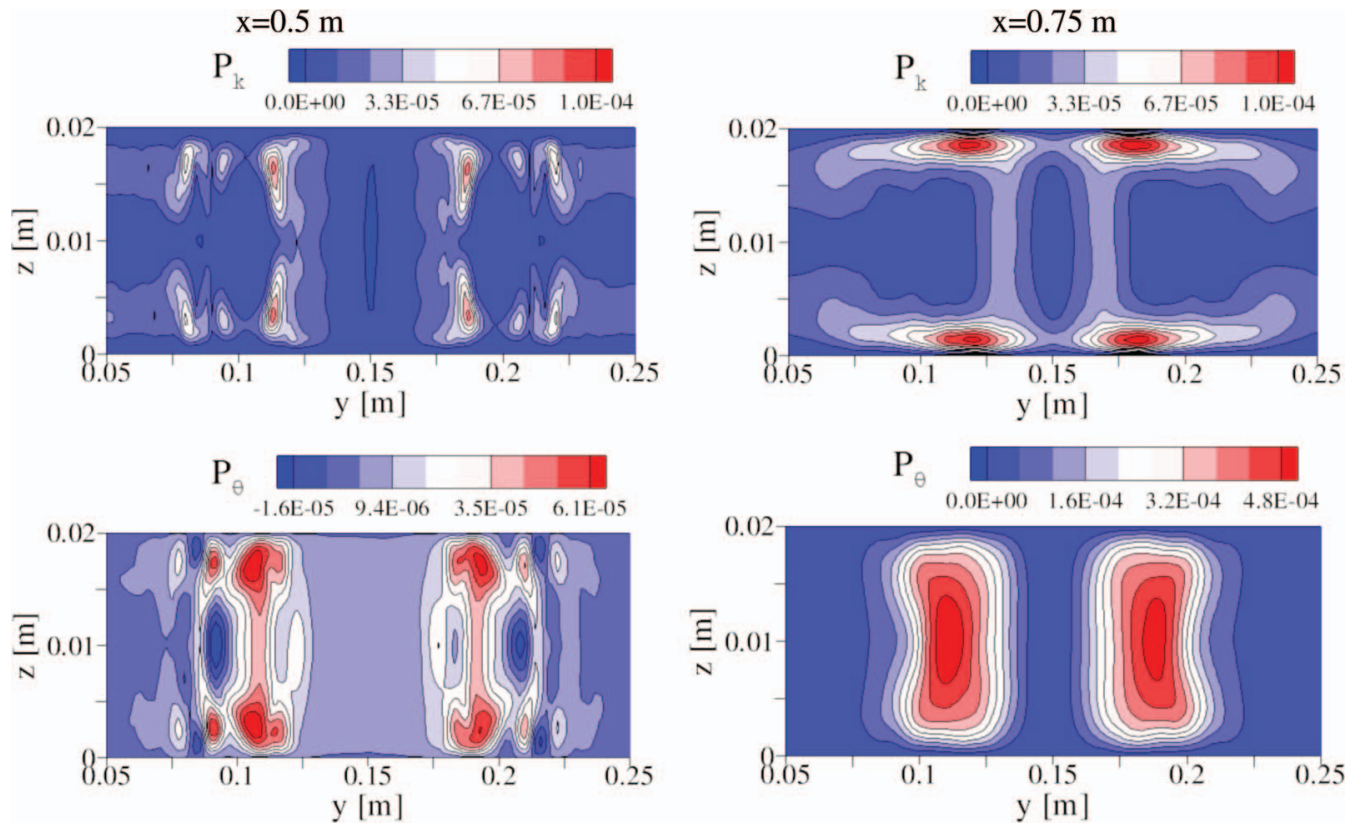

FIG. 8. Contours of the productions of the turbulent kinetic energy $\left(\mathrm{P}_{k}\right)\left(\right.$ top) and temperature variance $\left(\mathrm{P}_{\theta}\right)$ (bottom) in two vertical planes ( $\mathrm{x}=0.5 \mathrm{~m}$, left; $\mathrm{x}=0.75$, right) for the single-magnet configuration, $\mathrm{Re}=10^{3}, \mathrm{~N}=10$. Note that the vertical plane plots are not to scale. 

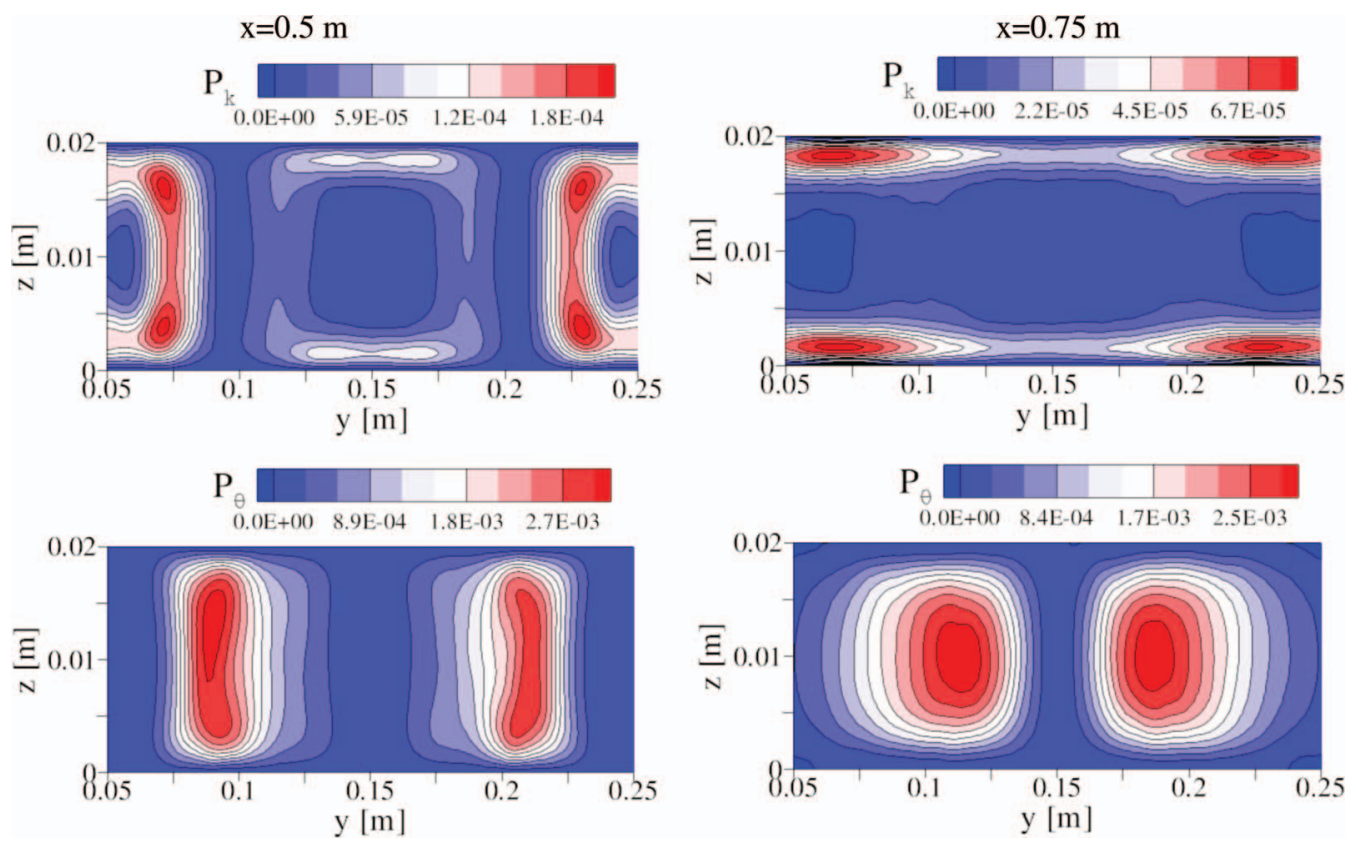

FIG. 9. Same as in previous figure, only for the two-magnet configuration, $\mathrm{N}=10$.

Fig. 7 (bottom). For the two-magnetic-dipoles case, local flow acceleration between magnetic dipoles prevents creation of strong temperature fluctuations along the magnetic edges, Fig. 7 (bottom/right).

The streamwise evolution of $P_{k}$ and $P_{\theta}$ are analyzed by plotting their contours in the characteristic vertical (y-z) planes, Figs. 8 and 9 . Here, six pairs of the $P_{k}$ are identified at $\mathrm{x}=0.5 \mathrm{~m}$ location, Fig. 8 (top/left). At the $\mathrm{x}=0.75 \mathrm{~m}$ location, merging of these structures takes place and the remaining four structures are generated, Fig. 8 (top/right). These structures are now located in the proximity of the horizontal walls and are symmetrically oriented with respect to the central vertical line $\mathrm{y}=0.15 \mathrm{~m}$. There is also a weaker interconnecting region in the central part of the channel. Imprints of these structures in the central horizontal plane are already discussed above, Fig. 7. For the two-magnetic-dipoles case, the initial orientation of the distinct $P_{k}$ regions at $\mathrm{x}=0.5 \mathrm{~m}$ shows that the turbulence is generated in the outside regions along the vertical lines $\mathrm{y}=0.075 \mathrm{~m}$ and $\mathrm{y}=0.225 \mathrm{~m}$, with a very strong interaction between the upper and lower wall regions, Fig. 9 (top/left). The intensity of $P_{k}$ is significantly smaller in the pockets in the proximity of the central vertical line $(\mathrm{y}=0.15 \mathrm{~m})$ with only a weak interconnecting region between upper and lower wall. In the $\mathrm{x}=0.75$ vertical plane, the contours of $P_{k}$ show that the wall regions are not interacting anymore and that turbulence is mainly present in the proximity of the horizontal walls, Fig. 9 (top/right).

The fluctuations of temperature develop and behave differently from the velocity fluctuations. For a single magnetic dipole case, six pairs of the $P_{\theta}$ pockets at the $\mathrm{x}=0.5 \mathrm{~m}$ are similar to $P_{k}$ distribution at the same location, but in contrast to the velocity fluctuations, the temperature fluctuations are also significant in the central part of the domain, Fig. 8 (bottom/left). Already at the $\mathrm{x}=0.75 \mathrm{~m}$ location, there is a strong dissimilarity between the velocity and temperature fluctuations, Fig. 8 (right). Now, the two distinct pockets of $P_{\theta}$ are presented with maximum values in the center of the domain, in contrast to $P_{k}$ where the maximum values are observed in the proximity of the horizontal walls, Fig. 8 (right). This is due to the different character of the wall-boundary conditions for instantaneous velocity and temperature. The zero velocity boundary condition imposes a strong gradient in the near-wall regions, which is not the case for the zero-gradient (adiabatic) condition for temperature. For two-magnetic-dipoles case, this dissimilarity between $P_{k}$ and $P_{\theta}$ is already present at $\mathrm{x}=0.5 \mathrm{~m}$ location, Fig. 9 (right). At the $\mathrm{x}=0.75 \mathrm{~m}$, the maximum values of the distinct $P_{\theta}$ regions are moved closer to the central vertical plane, $\mathrm{y}=0.15 \mathrm{~m}$.

The long-term averaged velocity, turbulent stress, temperature, and turbulent heat flux profiles extracted along the central $y=0.15 \mathrm{~m}$ line in the central $(\mathrm{z} / \mathrm{H}=0.5)$ horizontal $(x-y)$ plane are 

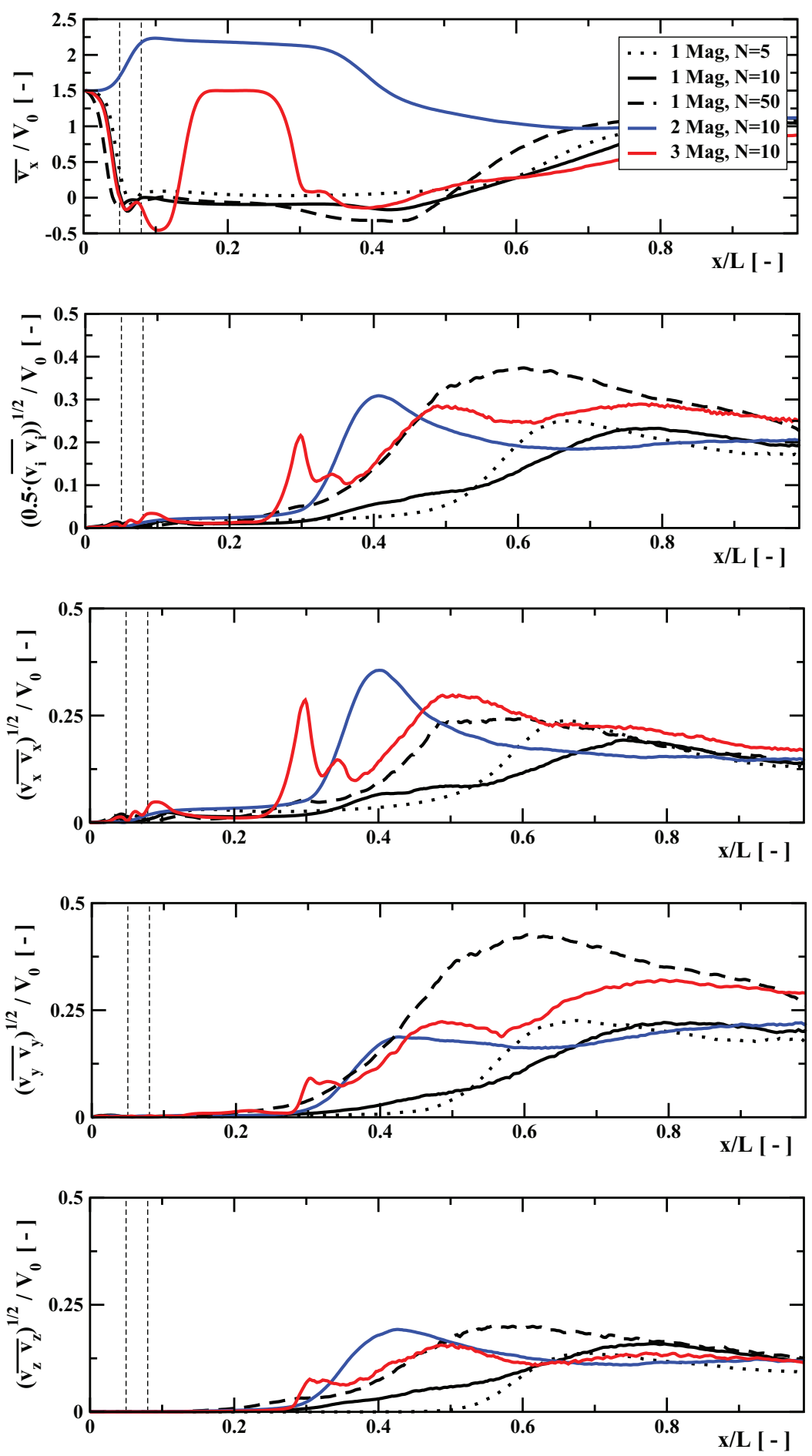

FIG. 10. (a)-(e) Profiles of the long-term averaged streamwise velocity, turbulent kinetic energy, and turbulent stresses in the central $(\mathrm{z} / \mathrm{H}=0.5)$ horizontal plane $(\mathrm{x}-\mathrm{y})$ and extracted along $\mathrm{y}=0.15 \mathrm{~m}$ for all configurations considered.

analyzed next, Figs. 10 and 11 . The mean streamwise velocity $\left(\overline{v_{x}}\right)$ profiles show a full range of different behaviors, Fig. 10(a). For a single magnetic dipole and $\mathrm{N}=5$, there is a small recirculative region just within the magnetic obstacle, while for $\mathrm{N}=10$, this recirculation region extends (although weak) till $\mathrm{x}=0.5 \mathrm{~m}$. For $\mathrm{N}=50$, a stronger recirculation $\left(\overline{v_{x}} / V_{0}\right.$ exceeds 0.25$)$ is located in 0.3 $\leq \mathrm{x} \leq 0.5 \mathrm{~m}$ region. In the case of two magnetic dipoles, no recirculation along the centerline 

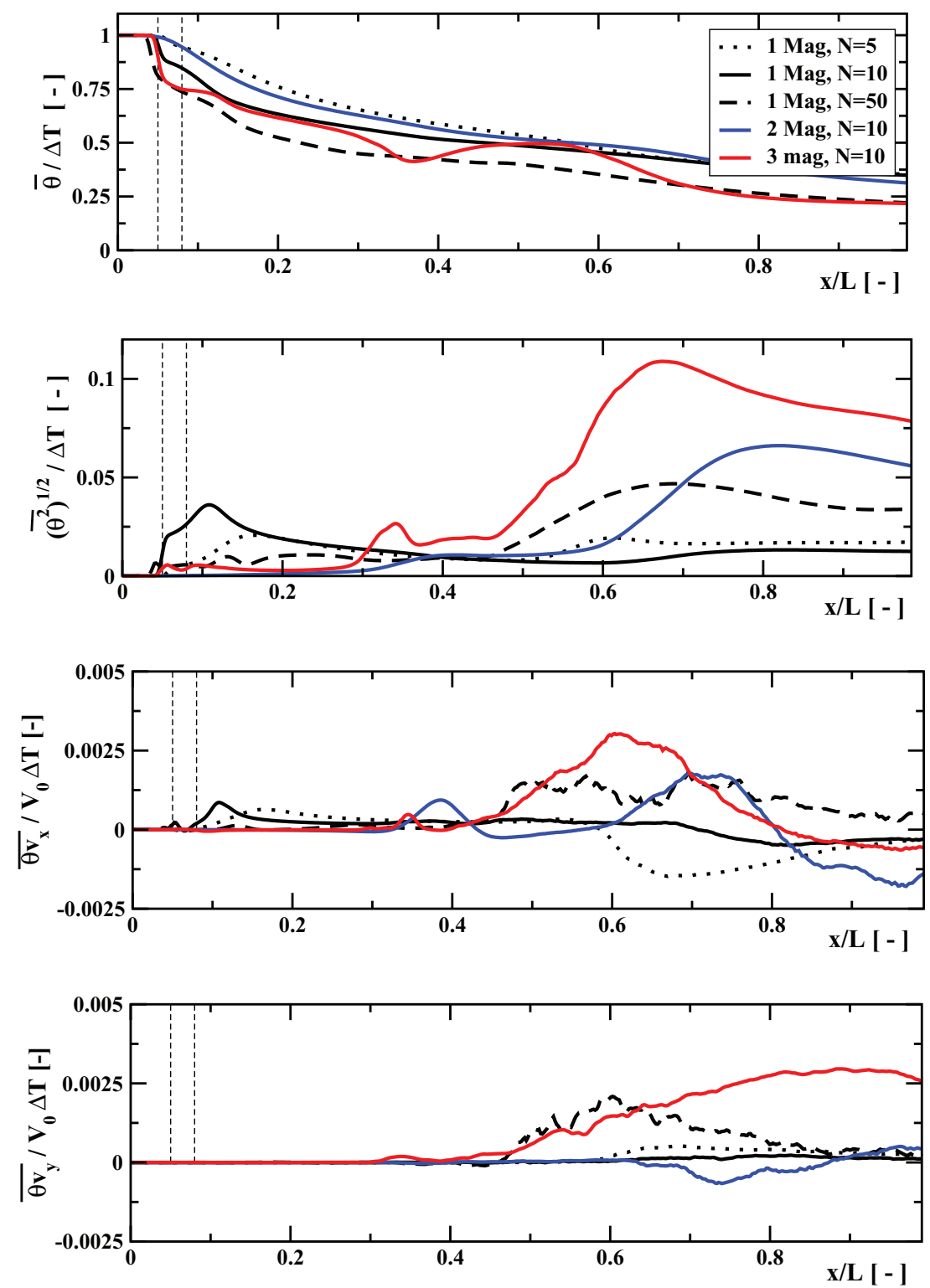

FIG. 11. (a)-(d) Same as in previous figure, only now for the temperature variance and turbulent heat flux components.

is observed. The incoming flow is pushed away from the staggered magnetic regions, resulting in strong flow acceleration along the centerline. The three-magnetic-dipoles case generates the most significant changes of the streamwise velocity along the centerline. The first flow reversal occurs in the core of the magnetic obstacle. Then, immediately after a short recovery, a sudden drop of $\overline{v_{x}} / V_{0}$ $=-0.5$ takes place at $\mathrm{x} / \mathrm{L}=0.1$. This is followed by a rapid flow acceleration and positive values of $\overline{v_{x}} / V_{0}$ of 1.5 are obtained at $\mathrm{x} / \mathrm{L}=0.15$. After this, flow is slowing down again and negative values of $\overline{v_{x}} / V_{0}$ are again obtained at $\mathrm{x} / \mathrm{L}=0.35$. After this, a monotonic flow recovery takes place.

For a single magnetic dipole, for different $\mathrm{N}$, distribution of the turbulent kinetic energy (TKE) exhibits relatively simple behavior with a monotonic increase, until the maximum value is reached, followed by a slow decrease, Fig. 10(b). The maximum values of TKE for $\mathrm{N}=5$ and 10 show similar values, although the location where TKE reaches its maximum are different $(\mathrm{x} / \mathrm{L}=0.65$, $0.75,0.6$ for $\mathrm{N}=5,10$, and 50, respectively). In the case of two magnetic dipoles the TKE profile is similar but its maximum value is reached already at $x / L=0.4$. The situation for the three magnetic 
dipoles is characterized by a multiple local maxima of TKE along the domain centerline. The first distinct maxima occurs at $x / L=0.3$ and is followed by the second at $x / L=0.5$ and the third at $x / L=0.75$. It is interesting to note that values of the second and third peaks of TKE are identical. To provide insights into the redistribution mechanism of the velocity fluctuations energy among the different components, profiles of the normal turbulent stress are analyzed next, Figs. 10(c)-10(e). For the single magnetic dipole, locations of the maximum values of the normal turbulent stress components are identical for a given value of $\mathrm{N}(\mathrm{x} / \mathrm{L}=0.65,0.75$, and 0.6 for $\mathrm{N}=5,10$, and 50 , respectively). While the streamwise turbulent stress is largest among the normal stress components for the single magnetic dipole and $\mathrm{N}=5$ and 10 , the situation is different for $\mathrm{N}=50$, where the spanwise component is the largest, Fig. 10(d). The vertical turbulent stress component $\left({\overline{v_{z}}}_{z}^{1 / 2} / V_{0}\right)$ is the smallest of the components due to blockage effects of the horizontal walls, Fig. 10(e). For the three magnetic dipoles, the multi-peak behavior is present for all normal stress components. An initial peak, though relatively small, occurs immediately after the magnetic core region and is followed by three additional distinct peaks, Fig. 10(c). These distinct peaks are gradually increasing for the spanwise component $\left({\overline{v_{y} v_{y}}}^{1 / 2} / V_{0}\right)$, Fig. 10(d). Both the streamwise and vertical components for all considered cases converge to similar values $\left(\bar{v}_{x} v_{x}^{1 / 2} / V_{0}={\overline{v_{z}}}_{z}^{1 / 2} / V_{0}=0.125\right)$ after $\mathrm{x} / \mathrm{L}$ $=0.85$, Figs. 10 (c) and $10(\mathrm{~d})$. The turbulence is still significantly anisotropic even after $\mathrm{x} / \mathrm{L}=0.85$, due to mostly dominant spanwise velocity contributions, Fig. 10(d).

The profiles of the mean temperature show significantly smaller variations for all cases considered, Fig. 11(a). This is due to the small value of the Prandtl number $(\operatorname{Pr}=0.022)$, which produces a strong diffusion. For intermediate and high values of $\mathrm{N}$, strongest gradients are observed within the magnetic core region. For the single magnetic dipole and $\mathrm{N}=50$, the temperature decrease starts even before the magnetic region - due to strong flow braking and build-up of the high-pressure region in the front of the magnetic obstacle. The profiles of temperature variance show significant dissimilarity to the distribution of the TKE, Fig. 11(b). For the single magnetic dipole case with $\mathrm{N}$ $=5$ and $\mathrm{N}=10$, the characteristic peak values are located in the near-wake of the magnetic core. With further increase of $\mathrm{N}=50$, the location of the maximum moves towards the far wake of the magnetic region, i.e., $x / L=0.65$. For the case of multiple dipoles, the temperature variance has two characteristic peaks in the distant wake behind the magnetic core - at $x / L=0.4$ and 0.8 for the two magnetic dipoles, and at $\mathrm{x} / \mathrm{L}=0.35$ and 0.65 for the three magnetic dipoles.

It is interesting to note the locations of countergradient diffusion, Figs. 11(a) and 11(c). The classical gradient type models assume that a scalar is transported down the gradient from regions with high scalar concentration to regions with low scalar concentration. For the case presented here, the scalar is temperature, and simple gradient diffusion in a model of turbulent heat flux assumes $\overline{\theta v_{x}}=-v_{t} / \operatorname{Pr}_{\mathrm{t}} \partial \bar{\theta} / \partial x$, where $\operatorname{Pr}_{\mathrm{t}}$ is the turbulent Prandtl number, and $v_{t}$ is the turbulent viscosity. Since both $\operatorname{Pr}_{\mathrm{t}}$ and $v_{t}$ are positive, the countergradient diffusion regions can be simply identified as regions where $\overline{\theta v_{i}}$ and $\partial \bar{\theta} / \partial x_{i}$ have the same sign. It can be seen from Fig. 11(a) that $\partial \bar{\theta} / \partial x<0$ for $0 \leq \mathrm{x} / \mathrm{L} \leq 1$ for all considered cases except for the case of three magnetic dipoles. For the latter one, $\partial \bar{\theta} / \partial x>0$ in $0.36 \leq x / L \leq 0.52$. For the single magnetic dipole and $N=5$, the countergradient heat flux occurs in $0.6 \leq \mathrm{x} / \mathrm{L} \leq 1$. With an increase of $\mathrm{N}=10$, this is reduced to $0.7 \leq \mathrm{x} / \mathrm{L} \leq 1$, and it totally diminishes for $\mathrm{N}=50$. In case of two magnetic dipoles, there are two segments with $\overline{\theta v_{x}}<0$, i.e., $0.45 \leq \mathrm{x} / \mathrm{L} \leq 0.6$ and $0.8 \leq \mathrm{x} / \mathrm{L} \leq 1$. There is a change of sign for the mean temperature gradient for three magnetic dipoles in $0.36 \leq \mathrm{x} / \mathrm{L} \leq 0.52$, i.e., $\partial \bar{\theta} / \partial x>0$. Now, the countergradient heat transfer takes place in $0.8 \leq \mathrm{x} / \mathrm{L} \leq 1$ as well as in $0.42 \leq \mathrm{x} / \mathrm{L} \leq 0.52$. The spanwise turbulent heat flux component is always positive, except for the case of two magnetic dipoles in $0.65 \leq \mathrm{x} / \mathrm{L} \leq 0.9$, Fig. 11(d). The spanwise turbulent heat flux is largest in the $0.4 \leq \mathrm{x} / \mathrm{L} \leq 0.9$ region, i.e., the region where fluctuations of the streamwise velocity are produces by a vortex shedding mechanism, as shown in Fig. 10(d). The only exception is for the case of three magnetic dipoles, where the streamwise heat flux shows significant contribution till the end of the domain, Fig. 11(d).

\section{E. Mixing efficiency}

To estimate which of considered configurations generated the best mixing, profiles of the nondimensional long-term averaged temperature $(\bar{\theta} / \Delta \theta)$ in the central vertical plane $(\mathrm{z} / \mathrm{H}=0.5)$ at 


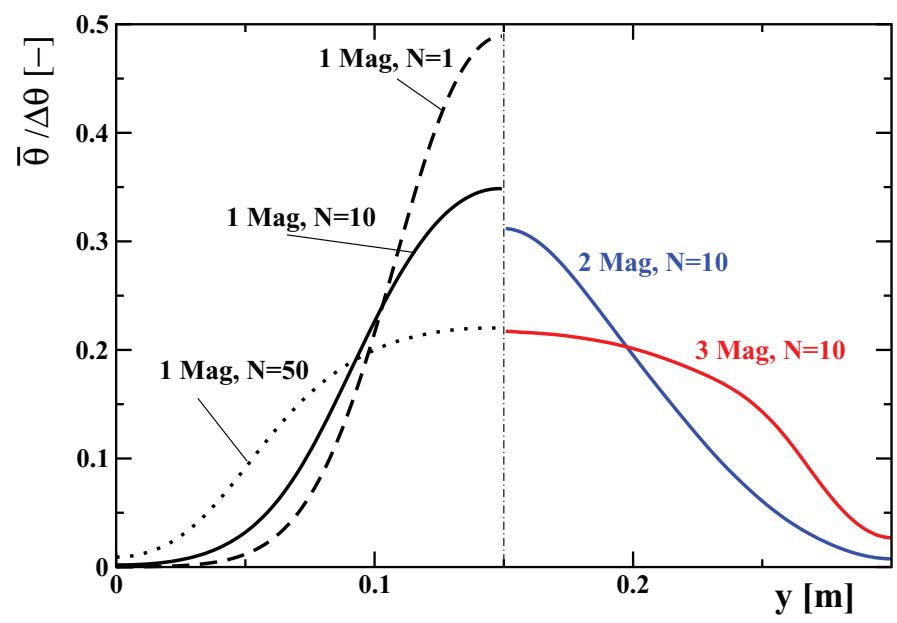

FIG. 12. The profiles of the non-dimensional long-term averaged temperature $(\bar{\theta} / \Delta \theta)$ in the central vertical plane $(\mathrm{z} / \mathrm{H}$ $=0.5)$ at the outlet of the domain $(\mathrm{x} / \mathrm{L}=1)$ for different magnetic-dipole configurations and different $\mathrm{N}$.

the outlet of the domain $(\mathrm{x} / \mathrm{L}=1)$ are shown in Fig. 12. For a single dipole configuration, with an increase of $\mathrm{N}$, the mixing efficiency also increases, but on a nonlinear manner. For identical values of the interactive parameter $\mathrm{N}$, two- and three-magnetic dipoles configuration show more efficient mixing. Note that a similar mixing efficiency is obtained with three-dipoles and $\mathrm{N}=10$ as for the single-dipole configuration at $\mathrm{N}=50$, indicating some interesting possibilities for application of multi-dipole configurations for practical situations.

\section{SUMMARY AND CONCLUSIONS}

We investigated flow and heat transfer of an electrically conducting fluid subjected to locally imposed magnetic fields (magnetic obstacles). The configurations with one, two, and three magnetic dipoles are investigated for different values of the interactive parameter $0 \leq \mathrm{N} \leq 50$ and a fixed fully developed laminar inflow at $\operatorname{Re}=10^{3}$. We solved iteratively a one-way coupled system of equations consisting of conservation of mass, momentum, and thermal energy (for pressure, velocity, and temperature, respectively) and Biot-Savart's law for spatial distributions of an external magnetic field, coupled through the Poisson equation for electric potential and Ohm's law for moving media for total electric charge density. Detailed insights into flow and heat transfer for both instantaneous and longterm time-averaged quantities are provided. It is shown that multi-magnetic dipoles configurations can bring additional alteration of the flow and consequently heat transfer (or mixing) compared with a single magnetic dipole configuration. This can be explored in practical applications where a local intensification of mixing or heat transfer is required. The power spectra density distributions are calculated for velocity, temperature, and electric potential at different monitoring locations in the central horizontal plane. The characteristic distinct frequencies are identified, confirming similarity of temporal dynamics between velocity and electric potential. The temperature spectra also show some additional distinct frequencies. The long-term averaged turbulence fields (for velocity and temperature) identified regions where the production of the turbulent kinetic energy and production of the temperature variance takes place. Despite the fact that temperature is a passive scalar, a strong dissimilarity between production mechanisms is observed. While the peaks of turbulent kinetic energy production are located in the proximity of horizontal walls, peaks of the production of the temperature variance are in the central region of the channel. The profiles of the turbulent stresses and turbulent heat fluxes in the central horizontal plane revealed a presence of anisotropic turbulence and countergradient diffusion of turbulent heat fluxes.

\footnotetext{
${ }^{1}$ Magneto-Hydrodynamics: Historical Evolution and Trends, edited by S. Molokov, R. Moreau, and H. K. Moffatt (Springer, Dordrecht, The Netherlands, 2007).
} 
${ }^{2}$ S. Gopalakrishnan, A. Thess, G. Weidmann, and U. Lange, "Chaotic mixing in a Joule-heated glass melt," Phys. Fluids 22(1), 013101 (2010).

${ }^{3}$ K. Cukierski and B. G. Thomas, "Flow control with local electromagnetic braking in continuous casting of steel slabs," Metall. Mater. Trans. B 39B, 94-107 (2007).

${ }^{4}$ A. Vire, B. Knaepen, and A. Thess, "Lorentz force velocimetry based on time-on-flight measurements," Phys. Fluids 22(12), 125101 (2010).

${ }^{5}$ M. Kirpo, S. Tympel, T. Boeck, D. Krasnov, and A. Thess, "Electromagnetic drag on a magnetic dipole near a translating conducting bar," J. Appl. Phys. 109, 113921 (2011).

${ }^{6}$ E. C. Brouillette and P. S. Lykoudis, "Magneto fluid mechanic channel flow. I. Experiment," Phys. Fluids 10, 995-1001 (1967).

${ }^{7}$ C. B. Reed and P. S. Lykoudis, "The effect of a transverse magnetic field on shear turbulence," J. Fluid Mech. 89, 147-171 (1978).

${ }^{8}$ Y. Shimomura, "Large eddy simulations of magnetohydrodynamic turbulent channel flow under a uniform magnetic field," Phys. Fluids 3(12), 3098-3106 (1991).

${ }^{9}$ D. Lee and H. Choi, "Magnetohydrodynamic turbulent flow in a channel at low magnetic Reynolds number," J. Fluid Mech. 439, 367-394 (2001).

${ }^{10}$ S. Kenjereš, K. Hanjalić, and D. Bal, "A direct-numerical-simulation-based second-moment closure for turbulent magnetohydrodynamic flows," Phys. Fluids 16(5), 1229-1241 (2004).

${ }^{11}$ T. Boeck, D. Krasnov, and E. Zienicke, "Numerical study of turbulent magnetohydrodynamic channel flow," J. Fluid Mech. 572, 179-188 (2007).

${ }^{12}$ L. Rossi, J. C. Vassilicos, and Y. Hardalupas, "Multiscale laminar flows with turbulentlike properties," Phys. Rev. Lett. 97(14), 144501 (2006).

${ }^{13}$ L. Rossi, J. C. Vassilicos, and Y. Hardalupas, "Electromagnetically controlled multi-scale flows," J. Fluid Mech. 558, 207-242 (2006).

${ }^{14}$ S. Lardeau, S. Ferrari, and L. Rossi, "Three-dimensional numerical simulation of electromagnetically driven multiscale shallow layer flows: Numerical modeling and physical properties," Phys. Fluids 20, 127101 (2008).

${ }^{15}$ L. Rossi, S. Bocquet, S. Ferrari, J. M. G. de la Cruz, and S. Lardeau, "Control of flow geometry using electromagnetic body forcing," Int. J. Heat Fluid Flow 30(3), 505-513 (2009).

${ }^{16}$ R. A. D. Akkermans, L. P. J. Kamp, H. J. H. Clercx, and G. J. F. van Heijst, "Three-dimensional flow in electromagnetically driven shallow two-layer fluids," Phys. Rev. E 82(2), 026314 (2010).

${ }^{17}$ M. Duran-Matute, R. R. Trieling, and G. J. F. van Heijst, "Scaling and asymmetry in an electromagnetically forced dipolar flow structure," Phys. Rev. E 83(1), 016306 (2011).

${ }^{18}$ S. Kenjereš, J. Verdoold, M. J. Tummers, K. Hanjalić, and C. R. Kleijn, "Numerical and experimental study of electromagnetically driven vortical flows," Int. J. Heat Fluid Flow 30(3), 494-504 (2009).

${ }^{19}$ S. Kenjereš, "Electromagnetic enhancement of turbulent heat transfer," Phys. Rev. E 78(6), 066309 (2008).

${ }^{20}$ S. Kenjereš, "Large eddy simulations of targeted electromagnetic control of buoyancy-driven turbulent flow in a slender enclosure," Theor. Comput. Fluid Dyn. 23(6), 471-489 (2009).

${ }^{21}$ S. Kenjereš, "Electromagnetically driven dwarf tornados in turbulent convection," Phys. Fluids 23(1), 015103 (2011).

${ }^{22}$ J. C. R. Hunt and G. S. S. Ludford, "Three-dimensional MHD duct flows with strong transverse magnetic fields: Part 1. Obstacles in a constant area channel," J. Fluid Mech. 33(4), 693-714 (1968).

${ }^{23}$ Y. B. Kolesnikov and A. B. Tsinober, "Two-dimensional turbulent flow behind a circular cylinder," Magnetohydrodynamics 3, 300-307 (1972).

${ }^{24}$ G. Mutschke, G. Gerbeth, V. Shatrov, and A. Tomboulides, "Two- and three-dimensional instabilities of the cylinder wake in an aligned magnetic field," Phys. Fluids 9(11), 1-3 (1997).

${ }^{25}$ G. Mutschke, G. Gerbeth, V. Shatrov, and A. Tomboulides, "The scenarios of three-dimensional instabilities of the cylinder wake in an external magnetic field: A linear stability analysis," Phys. Fluids 13(3), 723-734 (2001).

${ }^{26}$ B. Mück, C. Günther, U. Müller, and L. Bühler, "Three-dimensional MHD flows in rectangular ducts with internal obstacles," J. Fluid Mech. 418, 265-295 (2000).

${ }^{27}$ V. Dousset and A. Pothérat, "Numerical simulations of a cylinder wake under a strong axial magnetic field," Phys. Fluids 20, 017104 (2008).

${ }^{28}$ W. K. Hussam, M. C. Thompson, and G. J. Sheard, "Dynamics and heat transfer in a quasi-two-dimensional MHD flow past a circular cylinder in a duct at high Hartmann number," Int. J. Heat Mass Transfer 54, 1091-1100 (2011).

${ }^{29}$ S. Cuevas, S. Smolentsev, and M. A. Abdou, "On the flow past a magnetic obstacle," J. Fluid Mech. 553, 227-252 (2006).

${ }^{30}$ S. Cuevas, S. Smolentsev, and M. A. Abdou, "Vorticity generation in creeping flow past a magnetic obstacle," Phys. Rev. E 74(5), 056301 (2006).

${ }^{31}$ E. V. Votyakov, Y. Kolesnikov, O. Andreev, E. Zienicke, and A. Thess, "Structure of the wake of a magnetic obstacle," Phys. Rev. Lett. 98, 144504 (2007).

${ }^{32}$ E. V. Votyakov, E. Zienicke, and Y. B. Kolesnikov, "Constrained flow around a magnetic obstacle," J. Fluid Mech. 610, $131-156(2008)$.

${ }^{33}$ E. V. Votyakov and S. C. Kassinos, "On the analogy between streamlined magnetic and solid obstacles," Phys. Fluids 21(9), 097102 (2009).

${ }^{34}$ E. V. Votyakov and S. C. Kassinos, "Core of the magnetic obstacle," J. Turbul. 11(49), 1-15 (2010).

${ }^{35}$ A. Beltran, E. Ramos, S. Cuevas, and M. Brons, "Bifurcation analysis in a vortex flow generated by an oscillatory magnetic obstacle,” Phys. Rev. E 81, 036309 (2010).

${ }^{36}$ S. Kenjereš, S. ten Cate, and C. J. Voesenek, "Vortical structures and turbulent bursts behind magnetic obstacles in transitional flow regimes,” Int. J. Heat Fluid Flow 32(3), 510-528 (2011).

${ }^{37}$ S. Kenjereš and K. Hanjalić, "On the implementation of effects of Lorentz force in turbulence closure models," Int. J. Heat Fluid Flow 21(3), 329-337 (2000). 
${ }^{38}$ S. Kenjereš and K. Hanjalić, "Numerical simulation of magnetic control of heat transfer in thermal convection,” Int. J. Heat Fluid Flow 25(3), 559-568 (2004).

${ }^{39}$ S. Kenjereš and K. Hanjalić, "Numerical simulation of a turbulent magnetic dynamo," Phys. Rev. Lett. 98(10), 104501 (2007).

${ }^{40}$ S. Kenjereš and K. Hanjalić, “Numerical insights into magnetic dynamo action in a turbulent regime," New J. Phys. 9(306), 1-29 (2007).

${ }^{41}$ S. Kenjereš, "Hybrid simulations of two-way coupled turbulent magnetohydrodynamic flows," Int. J. Multiscale Comp. Eng. 7(6), 545-558 (2009).

${ }^{42}$ P. L. Roe, "Approximate Riemann solvers, parameter vectors, and difference schemes," J. Comput. Phys. 43, 357-372 (1981).

${ }^{43}$ Y. Cuypers, A. Maurel, and P. Petitheans, “Vortex bursts as a source of turbulence,” Phys. Rev. Lett. 91(19), 194502 (2003). 